\title{
Atomistic simulation of the structural and elastic properties of magnesite
}

\author{
ZI-JIANG LIU ${ }^{1,2,3, *}$, XIAO-WEI SUN ${ }^{2}$, TING SONG ${ }^{2}$, YUAN GUO ${ }^{1}$, CAI-RONG ZHANG ${ }^{4}$ and \\ ZHENG-RONG ZHANG ${ }^{1}$ \\ ${ }^{1}$ Department of Physics, Lanzhou City University, Lanzhou 730070, PR China \\ ${ }^{2}$ School of Mathematics and Physics, Lanzhou Jiaotong University, Lanzhou 730070, PR China \\ ${ }^{3}$ Key Laboratory of Atomic and Molecular Physics \& Functional Materials of Gansu Province, Lanzhou 730070, PR China \\ ${ }^{4}$ Department of Applied Physics, Lanzhou University of Technology, Lanzhou 730050, PR China
}

MS received 3 July 2014; accepted 9 March 2016

\begin{abstract}
Atomistic simulation was carried out to study the structural and elastic properties of $\mathrm{MgCO}_{3}$ magnesite within the pressure range of the Earth's mantle based on a novel force field. The lattice parameters and elastic constants as a function of pressure up to $150 \mathrm{GPa}$ are calculated. The results are in good agreement with the available experimental data and previous theoretical results, showing no phase transition over the pressure range of interest. We also found that magnesite exhibits a strong anisotropy throughout the lower mantle and that the nature of the anisotropy changes significantly with depth.
\end{abstract}

Keywords. Magnesite; structural property; elastic property; high pressure.

\section{Introduction}

Alkaline earth carbonates have been a subject of considerable interest in the Earth Sciences. They are the most abundant carbon-bearing species in the crust, representing a major reservoir for carbon within the Earth's mantle [1]. Among the alkaline earth carbonates, $\mathrm{MgCO}_{3}$ magnesite is one of the most extensively studied minerals. Numerous experimental [2-8] and theoretical $[2,9-11]$ studies have been dedicated to determining the stability of magnesite. However, it remains unclear whether magnesite is stable or not over the entire pressure and temperature range of the mantle.

Magnesite, being stable at ambient conditions $[3,6]$, can be transported deep into the Earth's mantle through the subduction of oceanic lithosphere [12]. Recent experimental and theoretical studies show that magnesite remains chemically stable at high pressure and high temperature conditions of the deep mantle, even though a number of structural transitions in $\mathrm{MgCO}_{3}$ have been reported [3,9]. For example, Fiquet et al [4] observed that magnesite undergoes a phase transition at $25 \mathrm{GPa}$. Isshiki et al [3] found that magnesite transforms to an unknown form at pressures above $115 \mathrm{GPa}$. Skorodumova et al [9] used ab initio calculations to determine that magnesite can transform into a pyroxene structure at $113 \mathrm{GPa}$. Oganov et al [10] performed simulations using the universal structure prediction programme (USPEX) code based on an evolutionary algorithm with $a b$ initio free energy as the fitness function and found that the post-magnesite phase (the $\mathrm{C} 222_{1}$ structure) is more stable than magnesite above $106 \mathrm{GPa}$. Further, using the same method and code between 82.4 and $138.1 \mathrm{GPa}$, Oganov et al [2] found that the most stable

\footnotetext{
*Author for correspondence (liuzj2000@126.com)
}

structure has a space group $C 2 / \mathrm{m}$. Recently, Panero and Kabbes [11] predicted that magnesite will undergo a structural transition to a pyroxene-like structure at 80-100 GPa.

To further understand the carbon cycle on a global scale, it is important to investigate the stability of magnesite under conditions similar to those of the Earth's mantle. To bring new insights on the stability and properties of magnesite at high pressure, we examined the structural properties of magnesite using interatomic potentials combined with a novel force field over the entire pressure range of the mantle.

Additionally, knowledge of the elasticity of magnesite and its pressure dependence helps us to understand seismological observations of the Earth's mantle. Empirical determination of elastic properties in extreme conditions is, however, not an easy task. The sound velocities and aggregate elastic properties of magnesite have only been measured at ambient conditions $[13,14]$. In a theoretical study, Brik [15] investigated the elastic properties of magnesite by means of the density functional theory in the generalized gradient approximation (GGA) and the local density approximation (LDA) at zero pressure. Many of the elastic properties of magnesite are still relatively poorly known.

\section{Computational method}

\subsection{Interatomic potential calculations}

Theoretical studies of materials require a model of the forces acting between the atoms in a solid. The atomistic simulation method uses interatomic potential functions to describe the total energy of a system in terms of atomic coordinates. Thus, the equilibrium positions of atoms or ions in a system 
are evaluated by minimizing the lattice energy until all the strains acting on the crystal are removed. The lattice energy can be defined as the sum of the electrostatic or Coulombic forces acting between atoms and the short-range repulsive forces produced by the overlap of nearest-neighbour electron clouds and Van der Waals forces. These short-range forces act between bonded and non-bonded atoms in the crystal, where non-bonded interactions can be effectively modelled by the Buckingham potential:

$$
U_{i j}^{\text {Buck }}=A_{i j} \exp \left(-r_{i j} / \rho_{i j}\right)-C_{i j} r_{i j}^{-6} .
$$

The parameters $A_{i j}$ and $\rho_{i j}$ describe the repulsion between two ions $i$ and $j$ at a separation distance of $r_{i j}$, and $C_{i j}$ is a term included to model dispersion. For covalent materials, the three-body potential represents the repulsion between bond pairs, or even occasionally between lone pairs. Hence, the form chosen is usually a harmonic one that penalizes deviation from the expected angle for the coordination environment, such as $120^{\circ}$ for a trigonal planar carbon atom:

$$
U_{i j k}^{\mathrm{Harm}}=0.5 k_{\theta}\left(\theta-\theta_{0}\right)^{2} .
$$

The four-body torsional potential is one that employs a harmonic potential to describe the out-of-plane bending mode of a central atom that has planar coordination geometry. It has been used in the modelling of the carbonate anion. The commonly used form is:

$$
U_{i j k l}=k_{2} d^{2}+k_{4} d^{4}
$$

To obtain the accurate values of the lattice dynamic properties and the elastic and dielectric constants, it is essential to include ionic polarization along with the short-range interactions. Polarization is included by using the shell model [16], which treats each ion in terms of a core connected via a harmonic spring constant to a massless having charge $Y$. The free-ion polarizability $\alpha$ may be written as:

$$
\alpha=\frac{Y^{2}}{k} \text {. }
$$

Finally, a Morse potential is used to describe the covalent bonding of the $\mathrm{C}-\mathrm{O}$ interaction in the carbonate group. Coulombic interactions are excluded within the molecular carbonate group.

The simulations in this work are performed in tandem with extending the general utility lattice program (GULP) bulk simulation code [17]. There have been numerous attempts to derive a satisfactory force field for the description of the polymorphs of magnesium carbonate. Of these, arguably the most accurate in terms of the description of the structure and physical properties of magnesite has been the potential of Rohl et al [18] and Austen et al [19] and the potential parameters are given in table 1. Energy minimizations are performed at constant pressure, from 0 to $150 \mathrm{GPa}$, allowing all individual ionic coordinates and lattice parameters to vary. The search of the local minima adopts the NewtonRaphson procedure, with the Broyden-Fletcher-GoldfarbShanno (BFGS) scheme to update the Hessian.

\subsection{Calculation of elastic constants}

Under a linear elastic deformation, the mechanical properties of crystals are described using Hooke's law:

$$
\sigma=C \varepsilon,
$$

where $\sigma$ is the stress, $\varepsilon$ the strain and $C$ the elastic constant

\begin{tabular}{|c|c|c|c|c|}
\hline Buckingham: $A \exp (-r / \rho)-C / r^{6}$ & $A(\mathrm{eV})$ & $\rho(\AA)$ & $C\left(\mathrm{eV} \AA^{6}\right)$ & Cutoff $(\AA)$ \\
\hline $\mathrm{O}_{\text {core }}-\mathrm{O}_{\text {core }}$ & 4030.300 & 0.245 & 0.000 & 2.5 \\
\hline $\mathrm{O}_{\text {shell }}-\mathrm{O}_{\text {shell }}$ & 64242.454 & 0.199 & 21.843 & 15.0 \\
\hline $\mathrm{Mg}-\mathrm{O}_{\text {core }}$ & 1039.590 & 0.289 & 0.000 & 10.0 \\
\hline $\mathrm{C}-\mathrm{Mg}$ & 26164795.400 & 0.120 & 0.000 & 10.0 \\
\hline Morse: $D\left(\left(1-\exp \left(-a\left(r-r_{0}\right)\right)\right)^{2}-1\right)$ & $D(\mathrm{eV})$ & $a\left(\AA^{-1}\right)$ & $r_{0}(\AA)$ & \\
\hline $\mathrm{C}-\mathrm{O}_{\text {core }}$ & 5.000 & 2.523 & 1.198 & 1 bond \\
\hline Spring: $0.5 k_{\mathrm{cS}} r^{2}$ & $k_{\mathrm{cs}}\left(\mathrm{eV} \AA^{-2}\right)$ & & & \\
\hline $\mathrm{O}_{\text {core }}-\mathrm{O}_{\text {shell }}$ & 52.740 & & & 0.6 \\
\hline Three-body: $0.5 k_{\theta}\left(\theta-\theta_{0}\right)^{2}$ & $k_{\theta}\left(\mathrm{eV} \mathrm{rad}^{-2}\right)$ & $\theta\left({ }^{\circ}\right)$ & & \\
\hline $\mathrm{O}_{\text {core }}-\mathrm{C}-\mathrm{O}_{\text {core }}$ & 1.799 & 120.00 & & Bonded \\
\hline Out of plane: $k_{2} d^{2}+k_{4} d^{4}$ & $k_{2}\left(\mathrm{eV} \AA^{-2}\right)$ & $k_{4}\left(\mathrm{eV} \AA^{-4}\right)$ & & \\
\hline $\mathrm{C}-\mathrm{O}_{\text {core }}-\mathrm{O}_{\text {core }}-\mathrm{O}_{\text {core }}$ & 8.689 & 360 & & Bonded \\
\hline Charges & (e) & & & \\
\hline $\mathrm{Mg}_{\text {core }}$ & +2.0 & & & No cutoff \\
\hline $\mathrm{C}_{\text {core }}$ & +1.343539 & & & \\
\hline $\mathrm{O}_{\text {core }}$ & +1.018487 & & & \\
\hline $\mathrm{O}_{\text {shell }}$ & -2.133 & & & \\
\hline
\end{tabular}
(matrix). Expressed in its components, it becomes:

$$
\sigma_{i j}=\sum_{k l} C_{i j k l} \varepsilon_{k l}
$$

Table 1. Potential terms and parameters of magnesite used in simulations. 
where $i, j, k, l=1,2,3,4, \sigma_{i j}$ is an element of the stress tensor, $\varepsilon_{k l}$ an element of the strain tensor and $C_{i j k l}$ are the elastic constants. In the above expression, we can use the more compact Voigt notation $(11=1,22=2,33=3,32$ or $23=4,31$ or $13=5,21$ or $22=6$ ), and the equation then becomes:

$$
\sigma_{\alpha}=\sum_{\beta} c_{\alpha \beta} \varepsilon_{\beta} .
$$

In Voigt notation, the strain tensor is:

$$
\bar{\varepsilon}=\left(\begin{array}{lll}
\varepsilon_{11} & \varepsilon_{12} & \varepsilon_{13} \\
\varepsilon_{21} & \varepsilon_{22} & \varepsilon_{23} \\
\varepsilon_{31} & \varepsilon_{32} & \varepsilon_{33}
\end{array}\right)=\left(\begin{array}{ccc}
\varepsilon_{1} & \frac{1}{2} \varepsilon_{6} & \frac{1}{2} \varepsilon_{5} \\
\frac{1}{2} \varepsilon_{6} & \varepsilon_{2} & \frac{1}{2} \varepsilon_{4} \\
\frac{1}{2} \varepsilon_{5} & \frac{1}{2} \varepsilon_{4} & \varepsilon_{3}
\end{array}\right) .
$$

According to Wallace [20], the internal energy of a crystal under strain, $\bar{\varepsilon}$ can be Taylor-expanded in powers of the strain tensor with respect to the initial energy of the unstrained crystal:

$E(V, \varepsilon)=E\left(V_{0}\right)+\sum_{\alpha: 1}^{6} \sigma_{\alpha \alpha}+\sum_{\alpha \beta: 1}^{6} c_{\alpha \beta} \varepsilon_{\alpha} \xi_{\alpha} \varepsilon_{\beta} \xi_{\beta}+\cdots$,

where $V$ and $V_{0}$ are the distorted and fully relaxed unit cell volumes, respectively. When introducing the Voigt notation, one has to remember that the elements of the strain tensor, $\varepsilon_{\alpha}$ are symmetric. To account for this, in the equation, the factor $\zeta_{\alpha}$ is equal to 1 if the Voigt index is 1,2 or 3 , and it is equal to 2 if the Voigt index is 4,5 or 6 . Therefore, the second-order elastic constants are related to the strain second derivatives of the total energy:

$$
c_{\alpha \beta}=\left.\frac{1}{V_{0}} \frac{\partial^{2} E}{\partial \varepsilon_{\alpha} \partial \varepsilon_{\beta}}\right|_{0} .
$$

The first-, third-, and higher-order terms in the equation are assumed to be zero, and this will be the case for pure linear elastic strains. Note that these terms may not be exactly zero in the calculations, but can be made close to zero with extremely small strains. Therefore, small elastic strains, $\varepsilon_{\alpha}$ can be applied to the fully relaxed unit cell lattice, and the elastic constants can be determined from the resulting change in energy.

\section{Results and discussion}

\subsection{Structural properties}

Magnesite has a hexagonal crystal system with rhombohedral symmetry and space group $R \overline{3} c$; the primitive cell contains two formula units. The calculations are performed using the primitive cell. The optimized structural parameters of magnesite are obtained by searching for the stable structure for a minimum total energy as mentioned in the method of calculation. The optimized lattice parameters, bond lengths and bond angles at zero pressure are shown in table 2 and compared with the experimental [4,6,7,21-24] and theoretical $[9,15,25,26]$ results. The calculated lattice parameters are in good agreement with the experimental data [4,6,7,21-24], whereas the LDA results (especially the $c$ constant) are somewhat underestimated and the GGA results are slightly overestimated. The calculated equation of state for magnesite is shown in figure 1, along with the previous experimental results $[7,21,23,24]$. The simulated equation of state is consistent with the experimental results. Magnesite compresses anisotropically, which is typical of all rhombohedral carbonates, with the $c$ axis being three times more compressible than the $a$ axis. Our calculated compression ratios differ slightly from those reported by Ross [7], but agree with the other experimental results $[21,23,24]$; the axial compressibilities $a / a_{0}$ and $c / c_{0}$ show a smooth continuous decrease with increasing pressure. Examination of these calculations using the GULP code reveals that there is a reasonably good agreement between the experimental and simulated structure, which shows that the present potential parameters can be used for further investigations at high pressures.

The structure of magnesite can be described as a distorted $\mathrm{NaCl}$ structure [27]. A convenient parameter to describe the degree of distortion from the $\mathrm{NaCl}$ structure is $t=4 a / \sqrt{2} c$, where $a$ and $c$ are the lattice parameters. In an ideal $\mathrm{NaCl}$ structure, $t=1$. The calculated $t$ value of magnesite is 0.881 at zero pressure. Structurally, the parameter $a$ is related to the $\mathrm{Mg}^{2+}-\mathrm{Mg}^{2+}$ distance and $c$ is related to the $\mathrm{Mg}^{2+}$

\begin{tabular}{|c|c|c|c|c|}
\hline Parameter & Present work & GGA & LDA & Experimental \\
\hline \multicolumn{5}{|l|}{ Lattice parameters } \\
\hline$a(\AA)$ & 4.647 & $\begin{array}{c}4.7119[15], \\
4.720[25]\end{array}$ & $\begin{array}{l}4.648[15], \\
4.635[25]\end{array}$ & $\begin{array}{c}4.635[4], 4.634[7], 4.635[21], \\
4.637[22], 4.628[23], 4.635[24]\end{array}$ \\
\hline$c(\AA)$ & 14.921 & $\begin{array}{c}15.4076[15], \\
15.331[25]\end{array}$ & $\begin{array}{l}14.775[15], \\
14.693[25]\end{array}$ & $\begin{array}{c}15.03[4], 15.178[7], 15.016[21], \\
15.023[22], 15.055[23], 15.024[24]\end{array}$ \\
\hline $\mathrm{V}\left(\AA^{3}\right)$ & 279.054 & $\begin{array}{c}289.8[9], 296.2[15] \\
295.8[25], 287.49[26]\end{array}$ & $276.4[15], 273.3[25]$ & $\begin{array}{c}279.2[6], 279.7[4], 279.28[7], 279.32[21], \\
279.503[22], 279.14[23], 279.55[24]\end{array}$ \\
\hline \multicolumn{5}{|l|}{ Bond lengths $(\AA)$} \\
\hline $\mathrm{C}-\mathrm{O}$ bond & 1.261 & $1.298[25]$ & $1.285[25]$, & $1.288[7], 1.283[22]$ \\
\hline $\mathrm{Mg}-\mathrm{O}$ bond & 2.115 & $2.149[25]$ & 2.087 [25], & $2.101[7], 2.105[22]$ \\
\hline Three $\mathrm{O}-\mathrm{Mg}-\mathrm{O}$ & $88.915,91.055$ & $88.263,91.737$ & $89.063,90.937$ & $88.252,91.752$ \\
\hline bond angles & 180.00 & $180[25]$ & $180[25]$ & $180[22]$ \\
\hline
\end{tabular}
$\mathrm{CO}_{3}^{2+}$ distance. The low $t$ values show that magnesite is less

Table 2. Lattice parameters, bond lengths and bond angles for magnesite. 


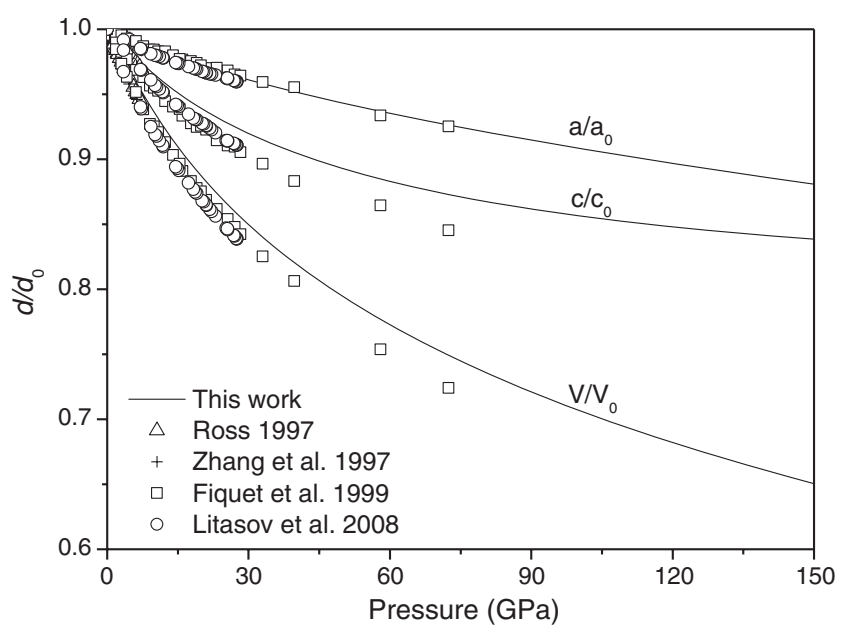

Figure 1. Pressure dependence of the axial compressibilities $a / a_{0}, c / c_{0}$ and the volume compressibility $V / V_{0}$ of magnesite.

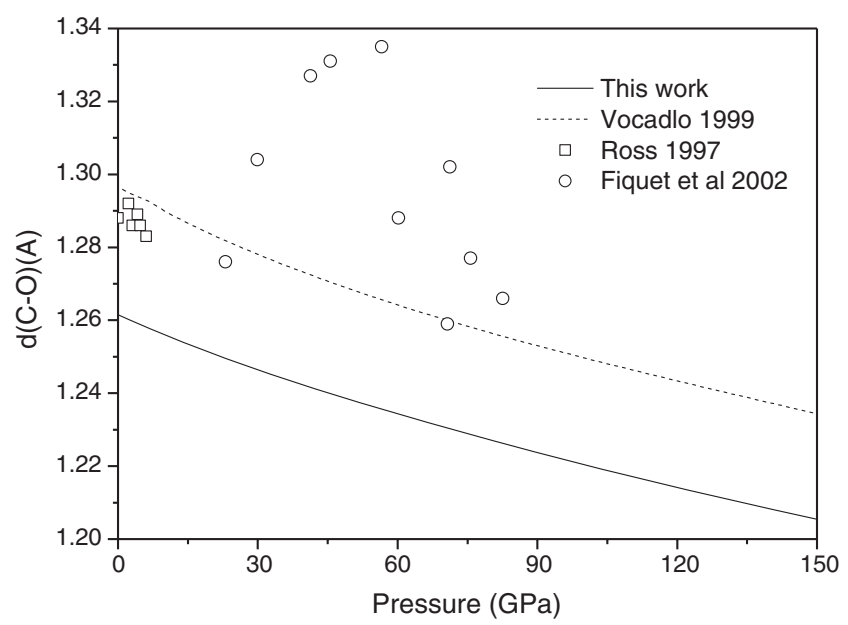

Figure 2. Pressure dependence of the $\mathrm{C}-\mathrm{O}$ bond length of magnesite.

compact along the $c$ axis than along the $a$ axis. This could partly explain the differences in relative compressibilities between the two axes.

The calculated bond lengths and angles for magnesite at zero pressure are summarized in table 2; results from available experimental [7,22] and theoretical [25] works are shown for comparison. The calculated $\mathrm{Mg}-\mathrm{O}$ bond length and the $\mathrm{O}-\mathrm{Mg}-\mathrm{O}$ bond angles are consistent with the experimental and theoretical data, while the $\mathrm{C}-\mathrm{O}$ bond length is slightly underestimated by $2 \%$. The variation of the calculated $\mathrm{C}-\mathrm{O}$ and $\mathrm{Mg}-\mathrm{O}$ bond lengths with pressure is shown in figures 2 and 3, respectively. Although, the calculated $\mathrm{C}-\mathrm{O}$ bond lengths differ slightly from the experimental results of Ross [7] in figure 2, the $\mathrm{C}-\mathrm{O}$ bond length is highly incompressible between 0 and $7 \mathrm{GPa}$; this effect is also observed in the calculations. Moreover, for the entire pressure range studied, the calculated results are essentially in parallel with those of Vočadlo [26], which were simulated using ab initio calculations based on density functional theory within the GGA. Our simulated results are $3 \%$ lower than those of Vočadlo at the

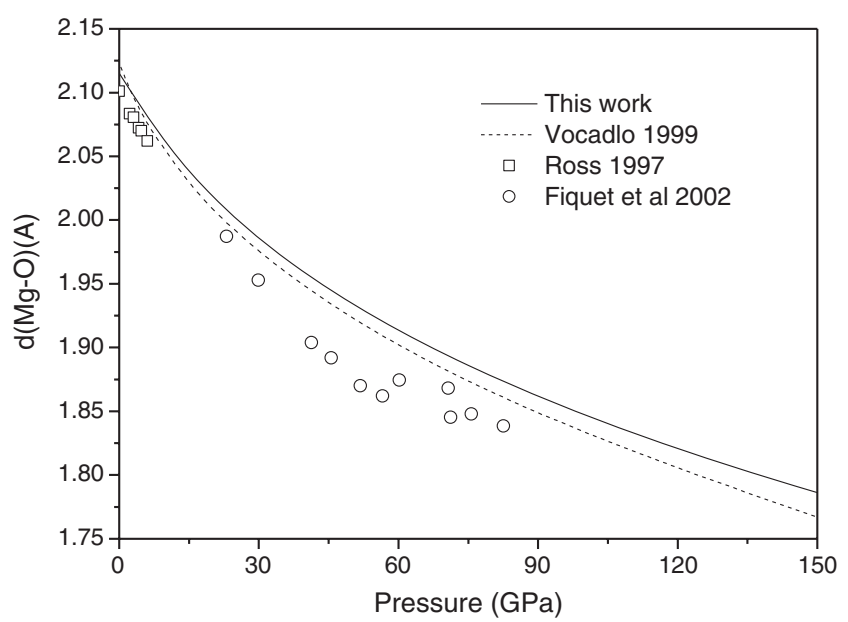

Figure 3. Pressure dependence of the $\mathrm{Mg}-\mathrm{O}$ bond length of magnesite.

same pressure, but do not agree with the experimental results of Fiquet et al [6] show that the $\mathrm{C}-\mathrm{O}$ bond length undergoes first a slight expansion with increasing pressure and then a strong compression above the threshold pressure of $60 \mathrm{GPa}$. The calculated $\mathrm{Mg}-\mathrm{O}$ bond lengths (figure 3) are in accordance with the experimental results of Ross [7] and the simulated results of Vočadlo [26]. All the three studies show that the $\mathrm{Mg}-\mathrm{O}$ bond is significantly compressible throughout the pressure range, while the observed results of Fiquet et al [6] indicate that the $\mathrm{Mg}-\mathrm{O}$ bonds cannot compress further above $60 \mathrm{GPa}$. As shown in figures 2 and 3, the calculated results do not indicate any obvious discontinuities in the internal structure through either the compression mechanism or discontinuous changes in bond lengths. Over the entire pressure range studied, the compression curves are smooth and continuous in all the cases. Hence, the present studies show no evidence of any high-pressure phase transition within the pressure range of the lower mantle, indicating that magnesite is stable throughout the lower mantle pressure range.

\subsection{Elastic properties}

The elastic constants determine the response of the crystal to external forces, as characterized by the bulk modulus, shear modulus, Young's modulus and Poisson's ratio, and play an important role in determining the strength of the materials. The elastic constants provide valuable information about the bonding characteristics between adjacent atomic planes and the anisotropic character of the bonding and structural stability. The elastic constants of the hexagonal phase magnesite are calculated by the method described above. A necessary condition for a crystal to be mechanically stable is that the elastic energy must be positive, or alternatively, its elastic stiffness matrix should satisfy the well-known Born stability criteria [28]. For hexagonal crystals, the requirement of mechanical stability leads to the following restrictions on its elastic constants [20]:

$c_{44}>0, \quad c_{11}>\left|c_{12}\right|, \quad\left(c_{11}+2 c_{12}\right) c_{33}>2 c_{13}^{2}$. 
The calculated elastic constants at zero pressure are tabulated in table 3 , where the available experimental results [13,14] and other theoretical results [15,29] are included. It shows that magnesite is mechanically stable in its crystal systems. The calculated elastic constants are in good agreement with the experimental results $[13,14]$ and the theoretical results [15]; as LDA tends to overbind the atoms in a crystal, the LDA results are larger than the GGA results. The singlecrystal elastic properties of mantle minerals at high pressure are essential for interpreting seismic wave velocities and their lateral variations. The single-crystal elastic constants of magnesite have not yet been measured in experiments at high pressure; the present work predicts the high-pressure elastic constants. Figure 4 shows that the predicted elastic constants increase smoothly and monotonically with increasing pressure.

The effective elastic moduli of polycrystalline aggregates are usually calculated by two approximations following Voigt [30] and Reuss [31], in which, uniform strain or stress are assumed, respectively, throughout the polycrystal. Hill [32] showed that the Voigt and Reuss averages are limits and suggested that the actual effective moduli can be approximated

Table 3. Elastic constants (GPa) of magnesite at zero pressure.

\begin{tabular}{lrrrrc}
\hline & $\begin{array}{c}\text { Present } \\
\text { work }\end{array}$ & $\begin{array}{r}\text { GGA } \\
{[15]}\end{array}$ & $\begin{array}{r}\text { LDA } \\
{[15]}\end{array}$ & $\begin{array}{c}\text { HF } \\
{[29]}\end{array}$ & Exp. \\
\hline$c_{11}$ & 247.7 & 223.1 & 263.9 & & \\
$c_{33}$ & 155.1 & 118.2 & 144.5 & 187 & $156[13], 159[14]$ \\
$c_{44}$ & 50.8 & 42.7 & 57.2 & & \\
$c_{66}$ & 77.2 & 83.5 & 96.0 & & \\
$c_{12}$ & 93.2 & 56.0 & 71.8 & & \\
$c_{13}$ & 77.7 & 35.9 & 66.9 & & \\
$c_{11}+c_{12}$ & 340.9 & 279.1 & 335.7 & 392 & $334[13], 361[14]$ \\
$B$ & 123.7 & 81.8 & 110.8 & 125 & $110[13], 117[14]$ \\
$G$ & 61.0 & 59.6 & 70.3 & & \\
\hline
\end{tabular}

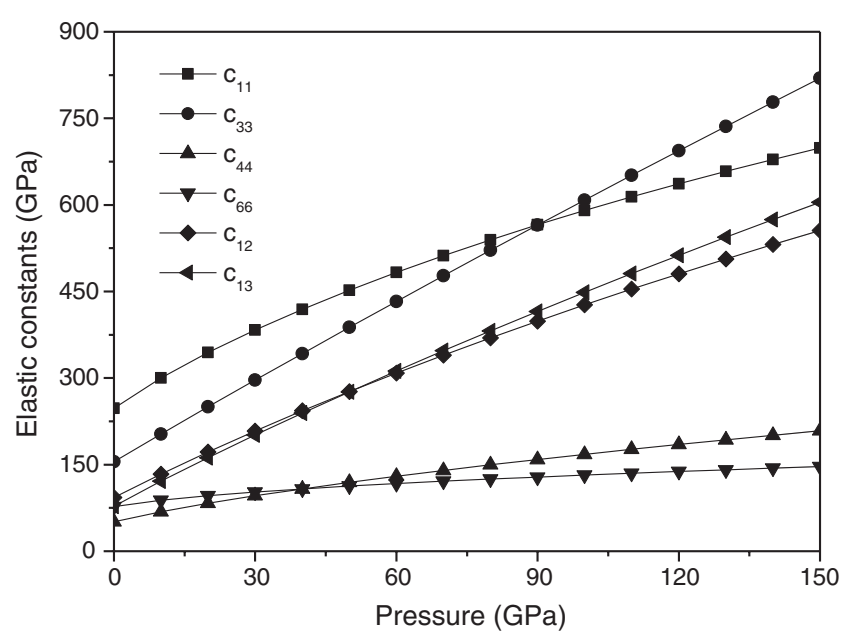

Figure 4. Pressure dependence of the elastic constants of magnesite. by the arithmetic mean of the two bounds. Based on the predicted elastic constants, the calculated bulk modulus $B$ and shear modulus $G$ of magnesite at zero pressure are given in table 3 . The calculated bulk modulus slightly overestimates the experimental results and is in excellent agreement with the theoretical results of Hartree-Fock (HF) [29].

To predict the brittle and ductile behaviour of solids, Pugh [33] introduced a simple relationship that empirically links the plastic properties of materials with their elastic moduli. The shear modulus $G$ represents the resistance to plastic deformation, while the bulk modulus $B$ represents the resistance to fracture. A high $B / G$ ratio is associated with ductility, whereas a low value corresponds to a brittle nature. The critical value that separates ductile and brittle materials is around 1.75 ; i.e., if $B / G>1.75$, the material behaves in a ductile manner; otherwise, the material behaves in a brittle manner. In the case of the hexagonal phase magnesite, the calculated value of $B / G$ is 2.03 , classifying this material as ductile.

Poisson's ratio $\sigma=(3 B-2 G) /(2(3 B+G))$ is associated with the volume change during uniaxial deformation. If $\sigma=$ 0.5 , no volume change occurs during elastic deformation. The calculated Poisson's ratio of magnesite at zero pressure is 0.29 ; the low $\sigma$ value means that a large volume change is associated with its deformation. In addition, $\sigma$ provides more information about the characteristics of the bonding forces than any of the other elastic constants [34]. It has been proven that $\sigma=0.25$ is the lower limit for central-force solids and 0.5 is the upper limit, which corresponds to infinite elastic anisotropy [35]. The low $\sigma$ value for magnesite indicates that the interatomic forces in the compound are non-central.

Knowledge of the elastic coefficients opens a way of evaluating the linear compressibilities. The ratio between the linear compressibility coefficients $k_{\mathrm{c}} / k_{\mathrm{a}}$ of a hexagonal crystal can be expressed as $k_{\mathrm{c}} / k_{\mathrm{a}}=\left(c_{11}+c_{12}-2 c_{13}\right) /\left(c_{33}-c_{13}\right)$. When $k_{\mathrm{c}} / k_{\mathrm{a}}>1$, the compressibility for magnesite along the $c$ axis is larger than the $a$ axis and the reverse is true when $k_{\mathrm{c}} / k_{\mathrm{a}}<1$. Our calculations show that the $k_{\mathrm{c}} / k_{\mathrm{a}}$ of magnesite is 2.39 at zero pressure, which means that the compressibility along the $c$ axis is much greater than the $a$ axis.

The evolution of elastic anisotropy of deep Earth minerals with increasing pressure and temperature is of fundamental importance for the understanding of the seismic anisotropy of the Earth's interior. To quantify the anisotropy of the elastic properties of magnesite, different criteria are employed. To investigate the contribution of the linear bulk modulus to the elastic anisotropy of magnesite, the bulk modulus along the crystal axes is calculated, and defined as $B_{i}=i d P / d i$, $i=a, c$ [36]. The calculated linear bulk modulus along the two directions $a$ and $c$ is seen in figure 5, where the linear bulk moduli of the two directions increase with rising pressure. $B_{\mathrm{c}}$ varies rapidly as the pressure increases and $B_{\mathrm{a}}$ increases comparatively slow with pressure. This indicates that the mechanical anisotropy of magnesite increases gradually with rising pressure. Moreover, it is interesting to note that the directional bulk modulus at high pressure is largest along the $c$ axis, and smallest along the $a$ axis, indicating 
that the compressibility along the $c$ axis is the smallest, while along the $a$ axis, it is largest.

The acoustic velocities can be obtained from elastic constants by the Christoffel equation $\left(c_{i j k l} n_{j} n_{k}-M \delta_{i l}\right) \mu_{i}=0$; the acoustic anisotropy can be described as $\Delta_{i}=M_{i}\left(n_{\mathrm{x}}\right) /$ $M_{i}(100)$. By solving the Christoffel equation for the hexagonal magnesite, the anisotropy of the compressional $(P)$ wave is obtained from $\Delta_{\mathrm{P}}=c_{33} / c_{11}$. The anisotropies of the polarized wave were perpendicular to the basal plane $(S 1)$ and the polarized basal plane $(S 2)$ are calculated as $\Delta_{S 1}=\left(c_{11}\right.$ $\left.+c_{33}-2 c_{13}\right) /\left(4 c_{44}\right)$ and $\Delta_{S 2}=c_{44} / c_{66}$, respectively. The calculated pressure dependences of the anisotropies $P, S 1$ and $S 2$ for the three types of elastic waves of magnesite are illustrated in figure 6 . While $P$ and $S 2$ increase with pressure, $S 1$ decreases sharply as the pressure increases (because the elastic constants $c_{11}$ and $c_{33}$ are affected by pressure). The anisotropy is dependent only on the symmetry of the crystal. The structure of the crystal changes under applied pressures

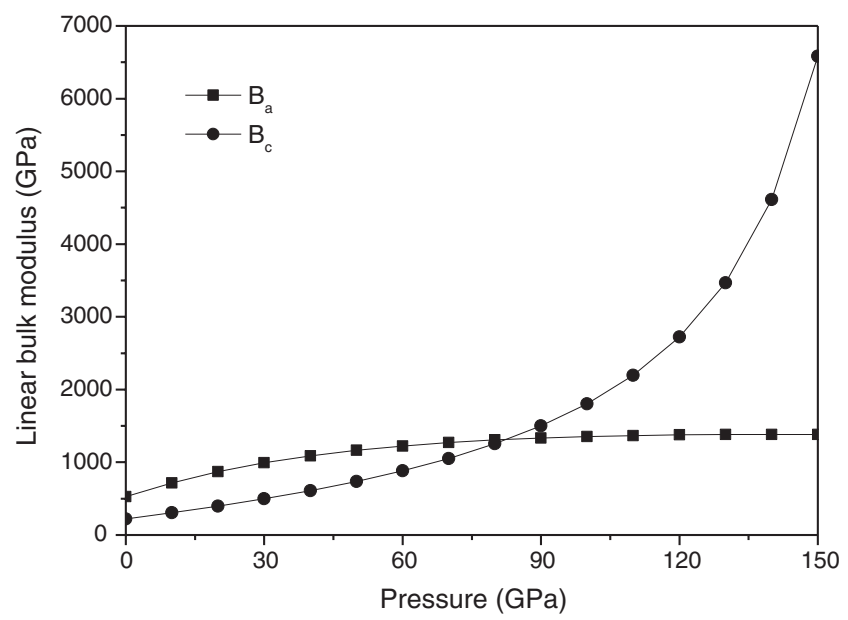

Figure 5. Pressure dependence of the linear bulk moduli of magnesite.

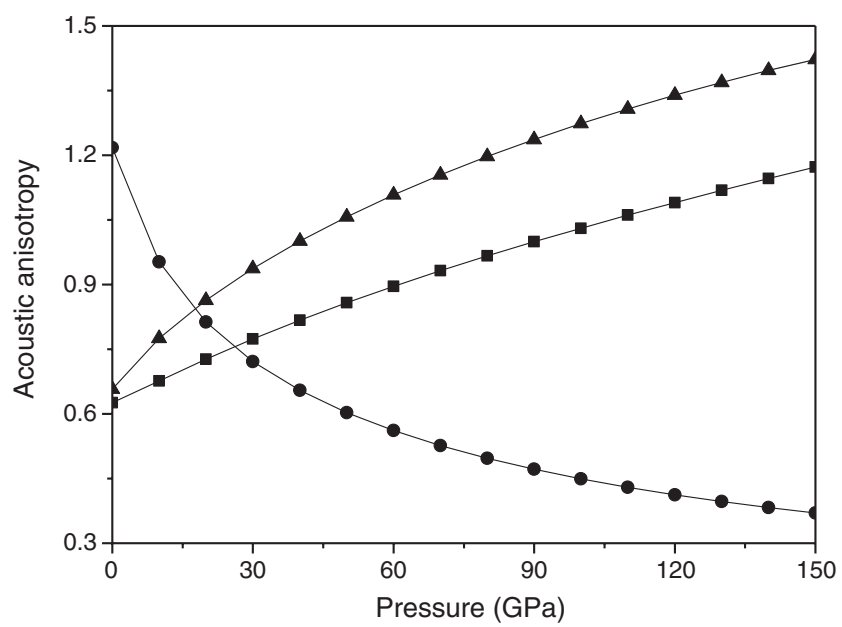

Figure 6. Pressure dependence of the acoustic anisotropy of magnesite. The squares, circles and triangles represent $P, S 1$ and $S 2$, respectively. because of the variations of $c / a$ at various pressures. Therefore, the elastic anisotropy is different due to the variations of the elastic constants with pressure.

The elastic anisotropy of crystals is also important for their applications. The elastic anisotropy of the low-symmetry crystal can be described by the percentage anisotropy in the compressibility $\left(A_{\mathrm{B}}\right)$ and shear $\left(A_{\mathrm{G}}\right)$ [37]. For $A_{\mathrm{B}}$ and $A_{\mathrm{G}}$, the values of 0 and 1 represent the elastic isotropy and the largest anisotropy, respectively. The pressure dependences of the percentage anisotropy in the compressibility and shear of magnesite are presented in figure 7. The calculated $A_{\mathrm{B}}$ and $A_{\mathrm{G}}$ indicate very little elastic anisotropy. At lower pressures, $A_{\mathrm{B}}$ and $A_{\mathrm{G}}$ have a trend of gradual decline with increasing pressure, then the curves rise when the pressure increases continually.

The anisotropy of the crystal can also be measured by the coefficients $A_{-}$and $A_{+}$calculated for each symmetry

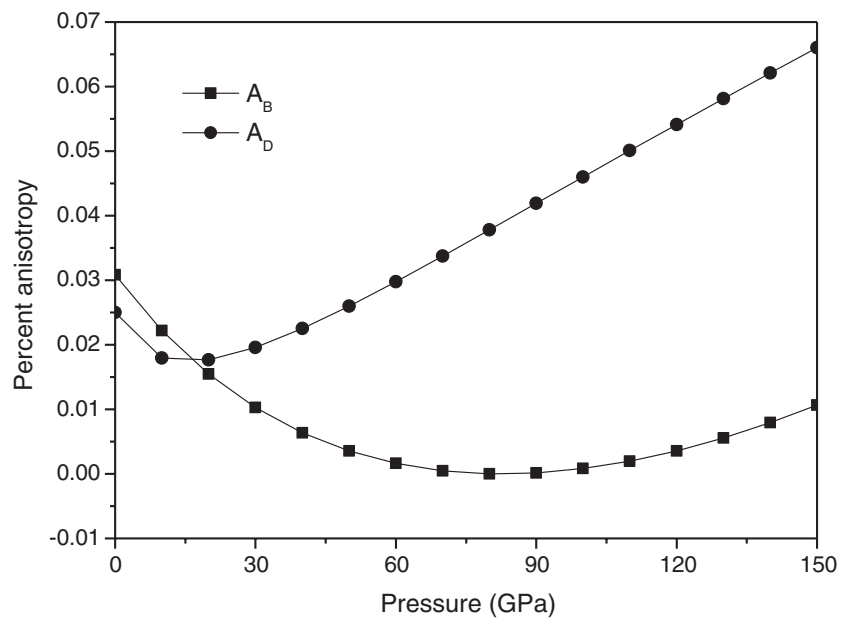

Figure 7. Pressure dependence of the percentage anisotropy of magnesite.

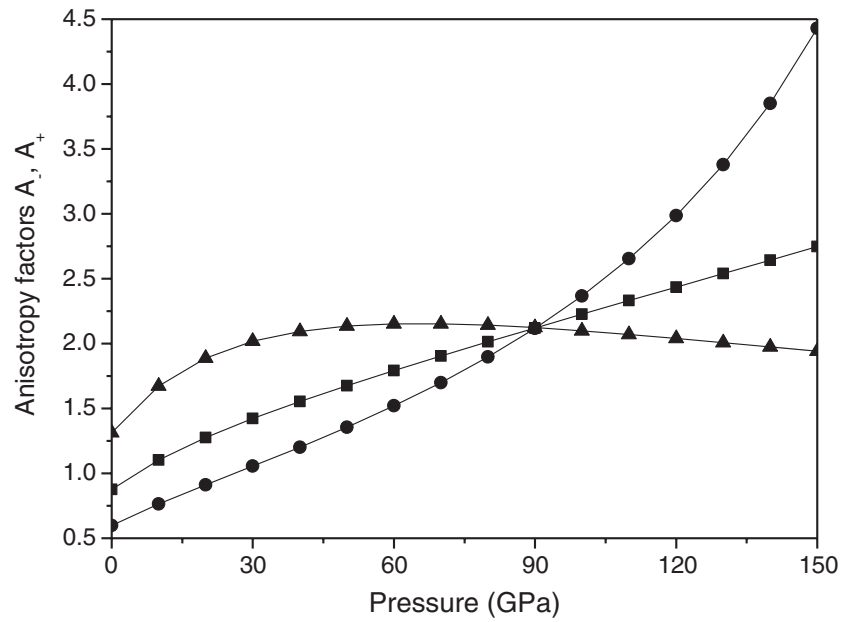

Figure 8. Pressure dependence of the anisotropy factors of magnesite. The squares, circles and triangles indicate $A_{-}^{[100]}, A_{+}^{[100],(010)}$ and $A_{+}^{[001],(010)}$, respectively. 
plane and axis; $[i j k]$ and (ijk) denote the symmetry axis and plane, respectively. These factors are derived from the elastic constants by the following simple relationships [38]

$$
\begin{aligned}
& A_{-}^{[100]}=c_{44}\left(c_{11}+2 c_{13}+c_{33}\right) /\left(c_{11} c_{33}-c_{13}^{2}\right), \\
& A_{+}^{[100],(010)}=2 c_{44} /\left(c_{11}-c_{13}\right), \\
& A_{+}^{[001],(010)}=2 c_{44} /\left(c_{33}-c_{13}\right) .
\end{aligned}
$$

The calculated anisotropy factors are shown in figure 8 . $A_{+}^{[100],(010)}$ varies rapidly as pressure increases and $A_{-}^{[100]}$ increases comparatively slow with pressure. However, $A_{+}^{[001],(010)}$ has an ascending trend at the lower pressure range, then turns to a slightly descending trend at the higher pressure range. This may indicate an increasing role of the anisotropic properties of magnesite in deep earth conditions, especially for magnesite with large-scale alignment of crystals caused by long-term laminar flows in the earth's interior.

\section{Conclusions}

The structural and elastic properties of magnesite were studied by atomistic simulation based on transferable empirical interatomic potentials combined with a novel force field over the entire pressure range of the mantle. The calculated structural parameters agree well with experimental and theoretical results. We found that the $\mathrm{CO}_{3}$ groups are rigid incompressible units, while the $\mathrm{Mg}-\mathrm{O}$ bond length undergoes significant compression. The calculation shows no phase transition over the entire pressure range studied, i.e., magnesite may be stable throughout the lower mantle. The predicted overall high-pressure elastic behaviour shows that the structure is quite anisotropic and that the anisotropy is strongly pressure-dependent. These results may have interesting implications for magnesite buried in the deep mantle in subduction zones and for the global carbon cycle; these may be revealed in future experimental and theoretical works.

\section{Acknowledgements}

This work was supported by the National Natural Science Foundation of China under Grant Nos 11464025, 11464027, 11164013 and 51562021, the program for Longyuan Youth Innovation Talents of Gansu Province of China, the Natural Science Foundation for Distinguished Young Scholars of Gansu Province under Grant No. 145RJDA323 and the Natural Science Foundation of Gansu Province of China under Grant Nos 1208RJZA242 and 1310RJZA025.

\section{References}

[1] Williams Q, Collerson B and Knittle E 1992 Am. Mineral. 77 1158

[2] Oganov A R, Ono S, Ma Y, Glass C W and Garcia A 2008 Earth Planet. Sci. Lett. 27338
[3] Isshiki M, Irifune T and Hirose K 2004 Nature 42760

[4] Fiquet G, Guyot F and Itie L P 1994 Am. Mineral. 7915

[5] Fiquet G, Richet P and Montagna G 1999 Phys. Chem. Miner. 27103

[6] Fiquet G, Guyot F, Kunz M, Matas J, Andrault D and Hanfland M 2002 Am. Mineral. 871261

[7] Ross N L 1997 Am. Mineral. 82682

[8] Gillet P 1993 Am. Mineral. 781328

[9] Skorodumova N V, Belonoshko A B, Huang L, Ahuja R and Johansson B 2005 Am. Mineral. 901008

[10] Oganov A R, Glass C W and Ono S 2006 Earth Planet. Sci. Lett. 24195

[11] Panero W R and Kabbes J E 2008 Geophys. Res. Lett. 35 L14307

[12] Seto Y, Hamane D, Nagai T and Fujino F 2008 Phys. Chem. Miner. 35223

[13] Humbert P and Plicque F 1972 C. R. Acad., Sci. Paris Ser. B 275391

[14] Dandekar D P and Ruoff A L 1968 J. Appl. Phys. 396004

[15] Brik M G 2011 Physica B 4061004

[16] Dick B G and Overhauser A W 1958 Phys. Rev. 11290

[17] Gale J D 1997 J. Chem. Soc., Faraday Trans. 93629

[18] Rohl A L, Wright K and Gale J D 2003 Am. Mineral. 88921

[19] Austen K F, Wright K, Slater B and Gale J D 2005 Phys. Chem. Chem. Phys. 74150

[20] Wallace D C 1972 Thermodynamics of crystals (New York: Wiley).

[21] Zhang J, Martinez I, Guyot F, Gillet P and Saxena S K 1997 Phys. Chem. Miner. 24122

[22] Oh K D, Morikawa H, Iwai S I and Aoki H 1973 Am. Mineral. 581029

[23] Fiquet G and Reynard B 1999 Am. Mineral. 84856

[24] Litasov K D, Fei Y, Ohtani E, Kuribayashi T and Funakoshi K 2008 Phys. Earth Planet. Int. 168191

[25] Hossain F M, Dlugogorski B Z, Kennedy E M, Belova I V and Murch G E 2010 Solid State Commun. 150848

[26] Vočadlo L 1999 Am. Mineral. 841627

[27] Wells A F 1984 Structural inorganic chemistry (Oxford, England: Clarendon).

[28] Fedorov F I 1968 Theory of elastic waves in crystals (New York: Plenum Press).

[29] Catti M, Pavese A, Dovesi R and Saunders V R 1993 Phys. Rev. B 479189

[30] Voigt W 1928 Lehrbuch de Kristallphysik (Leipzig: Terubner).

[31] Reuss A 1929 Z. Angew. Math. Mech. 949

[32] Hill R 1952 Proc. Phys. Soc., London Sect. A 65349

[33] Pugh S F 1954 Philos. Mag. 45823

[34] Köster W and Franz H 1961 Metall. Rev. 61

[35] Ledbetter M H 1983 in (eds) R P Reed and A F Clark (Metals Park, OH: American Society for Metals)

[36] Ravindran P, Fast L, Korzhavyi P A, Johansson B, Wills J and Eriksson O 1998 J. Appl. Phys. 844891

[37] Chung D H and Buessem W R 1967 J. Appl. Phys. 382010

[38] Lau K and McCurdy A K 1998 Phys. Rev. B 588980 